EASD

Procedia
EURODYN 2020

XI International Conference on Structural Dynamics M. Papadrakakis, M. Fragiadakis, C. Papadimitriou (eds.) Athens, Greece, 23-26 November 2020

\title{
RELIABILITY ASSESSMENT OF THE DYNAMIC BEHAVIOR OF HIGH-SPEED RAILWAY BRIDGES USING FIRST ORDER RELIABILITY METHOD
}

\author{
Reza Allahvirdizadeh ${ }^{1}$, Andreas Andersson $^{2}$, and Raid Karoumi ${ }^{3}$ \\ ${ }^{1}$ Division of Structural Engineering and Bridges, KTH Royal Institute of Technology, \\ Stockholm, Sweden \\ e-mail: reza.allahvirdizadeh@byv.kth.se \\ ${ }^{2}$ Division of Structural Engineering and Bridges, KTH Royal Institute of Technology, \\ Stockholm, Sweden \\ Swedish Transport Administration, Solna, Sweden \\ e-mail: andreas.andersson@byv.kth.se \\ ${ }^{3}$ Division of Structural Engineering and Bridges, KTH Royal Institute of Technology, \\ Stockholm, Sweden \\ e-mail: raid.karoumi@byv.kth.se
}

Keywords: High-speed railway bridges, Bridge dynamics, Structural reliability, First order reliability methods, Running safety, Serviceability limit state.

\begin{abstract}
The operational speed of the trains is intended to be significantly increased forthcoming; which consequently questions the safety level of the current design concepts concerning different performance limits. Thus, the reliability of the simply supported single-span bridges is assessed in the current article adopting the first-order reliability method (FORM) approach. In this regard, the dynamic response of the aimed bridges is investigated under the passage of a series of moving loads using available closed-form solutions in the literature. Hereof, axle load, car body/train configuration, flexural rigidity, damping, mass and model uncertainties are considered as random variables; while traintrack-bridge interactions are neglected and the procedure is repeated for a wide range of span lengths and train velocities. Then, the safety index corresponding to each case is evaluated by considering running safety as the limit state function; where, the bridge deck vertical acceleration is taken as the capacity of the system. The outcomes are presented as average probability of exceeding the limit state versus train speed and categorized based on span lengths.
\end{abstract}




\section{INTRODUCTION}

Former studies have shown that ballast instability is the dominant design criteria of high-speed railway bridges in comparison to the contact loss or excessive deflections. The latter statement is particularly validated for short to medium span bridges using numerical simulations $[1,2]$. It was observed that this phenomenon may occur at high vertical accelerations leading the interlock between grains to be lost or the lateral resistance of sleeper to be reduced. Its possibility of occurrence significantly increases under resonance conditions; which may occur due to high speed and repeated action of the axle loads [3].

In this regard, Zacher and Baeßler [4] conducted a shaking table test; in which they observed that the lateral resistance of the sleeper was significantly reduced at vertical accelerations about $7 \mathrm{~m} / \mathrm{s}^{2}$. The obtained outcomes revealed that the proposed vertical acceleration limit in EN-1990-A2 [5], i.e. $3.5 \mathrm{~m} / \mathrm{s}^{2}$ for ballasted tracks comply with a safety factor of 2 . Therefore, further researches particularly reliability assessments may be conducted to evaluate the achieved safety level by following current regulations. Those investigations may lead to more reliable design thresholds.

Generally, probability of failure/violation $\left(p_{f}\right)$ of limit state (performance) function can be estimated by calculating reliability integral as follows [6]:

$$
p_{f}=P[R-S \leq 0]=P[G(\mathbf{X}) \leq 0]=\int \ldots \int_{G(X) \leq 0} f_{X}(\mathbf{x}) d \mathbf{x}
$$

Where, $G(\mathbf{X})$ is the limit state function (safety margin) representing capacity (resistance - $R$ ) minus demand (load effects - $S$ ) and $f_{X}(\mathbf{x})$ is the joint probability density function of all random (basic) variables.

The aforesaid integration cannot be calculated in real (complicated) problems; in which contributing random variables present an $n$-dimensional hyperspace. Thus, practical solutions have been developed to either numerically evaluate the integral (such as Monte Carlo simulations) or approximate it (e.g. First Order Reliability Method - FORM). The current article adopted FORM to evaluate the probability of ballast instability in simply supported bridges under the passage of high-speed trains.

Within the FORM method, the safety level is expressed as a reliability index $(\beta)$; which is the shortest distance from the origin to the desired limit state function (see Figure 1). Then, the probability of failure can be estimated as Eq. 2:

$$
p_{f} \approx \Phi(-\beta)
$$

Where $\Phi$ is the standard Normal (Gaussian) distribution function.

Generally, FORM considers the linear approximation of the limit state function using Taylor series expansion around the most probable point (MPP) or checking point (shown as $u^{*}$ in Figure 1); which its surrounding area has the highest contribution on the failure probability. Detailed information regarding FORM can be found in $[6,7]$. Therefore, its outcome would depend on the used formulation defining failure surface; which is contrary to invariant problem. Thus, considered random variables and consequently, the limit state function should be transformed to standard Normal space using Hasofer-Lind transformation (Eq. 3).

$$
U_{i}=\frac{X_{i}-\mu_{X_{i}}}{\sigma_{X_{i}}}
$$


Where, $X_{i}$ is the basic random variable with first two moments of $\mu_{X_{i}}$ and $\sigma_{X_{i}}$ and $U_{i}$ is the transformed random variable.

Furthermore, the obtained direction cosines $(\boldsymbol{\alpha})$ can be used as a sensitivity of the transformed limit state function to the random variables. In this study, the omission sensitivity factor $\left(\gamma_{i}\right)$ is employed in addition to the direction cosines; which presents the relative importance of the random variable on reliability index if it was considered as deterministic. It is defined as the ratio of the reliability index when the $i^{\text {th }}$ random variable is deterministic $\left(\beta_{i}^{f}\right)$ to the reliability index when all variables are stochastic [8]. For independent random variables it would be as follows:

$$
\gamma_{i}=\frac{\beta_{i}^{f}}{\beta}=\frac{1}{\sqrt{1-\alpha_{i}^{2}}}
$$

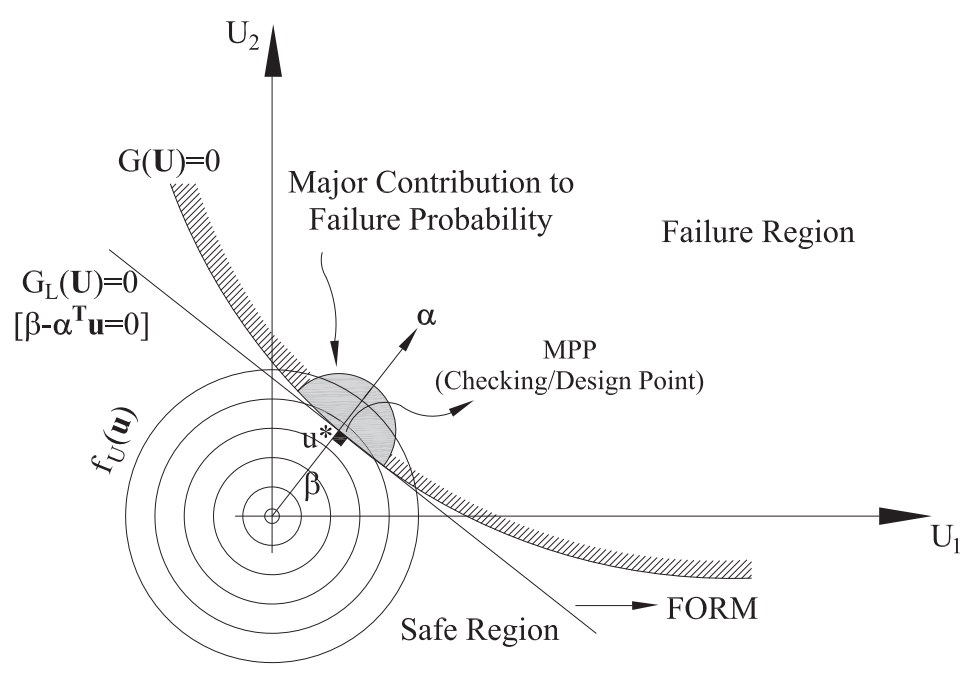

Figure 1: Schematic concept of the FORM $[6,7]$

\section{CONSIDERED ANALYTICAL SOLUTION}

Detailed investigation of the dynamic behavior of railway bridges can be achieved by modeling bridge structure, boundary conditions (soil-structure interaction - SSI), ballast, track, rail, train and their interactions (train-track-bridge interaction - TTBI). Schematic view of such a general model is shown in Figure 2a. In spite of providing detailed insight regarding dynamic response, the general model requires considering many random variables; which significantly increases computational costs of reliability evaluations. Furthermore, the probability characteristics of interaction- and boundary condition related variables are not well exploited. Thus, a simplified model including passage of a series of moving loads along a simply supported beam is taken into account here (see Figure $2 \mathrm{~b}$ ).

A close-form solution has been previously presented by $[9,10]$ to calculate beam deflection under such loading scenario. Therefore, the second derivative of the proposed solution is derived here to estimate the vertical acceleration of the bridge. Those equations are presented in Eqs. (5) to (10).

$$
\ddot{u}(t)=\sum_{n=1}^{\infty} \ddot{q}_{n}(t) \phi_{n}(x)=\sum_{n=1}^{\infty} \ddot{q}_{n}(t) \sin \left(\frac{n \pi x}{L}\right)
$$




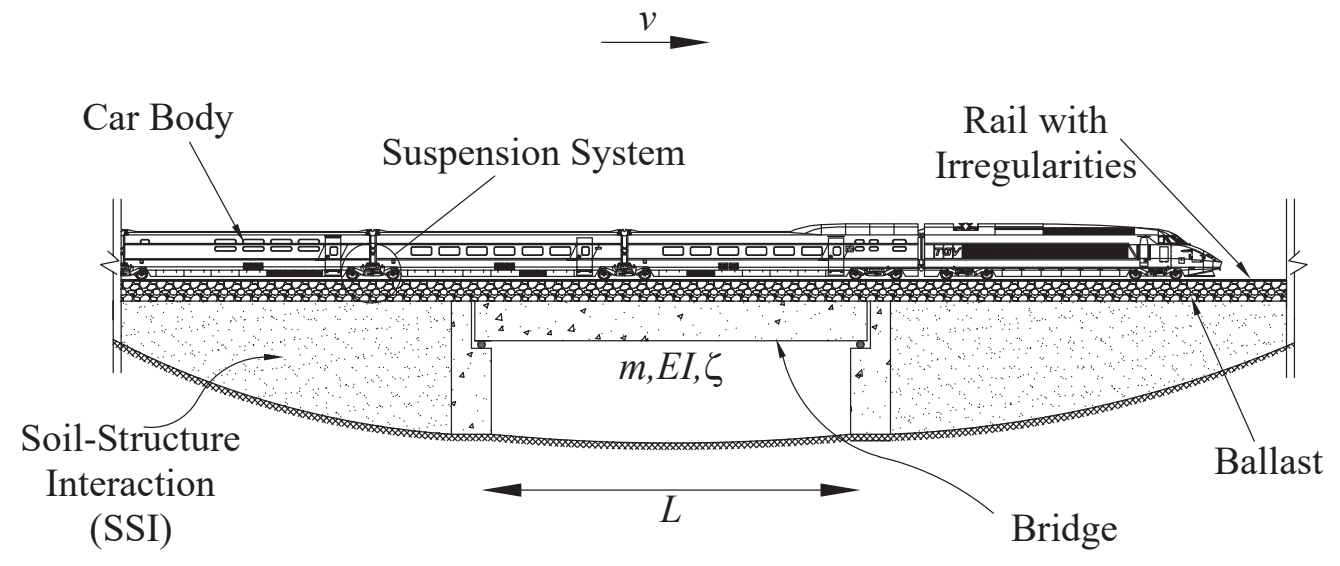

(a) General model with TTBI

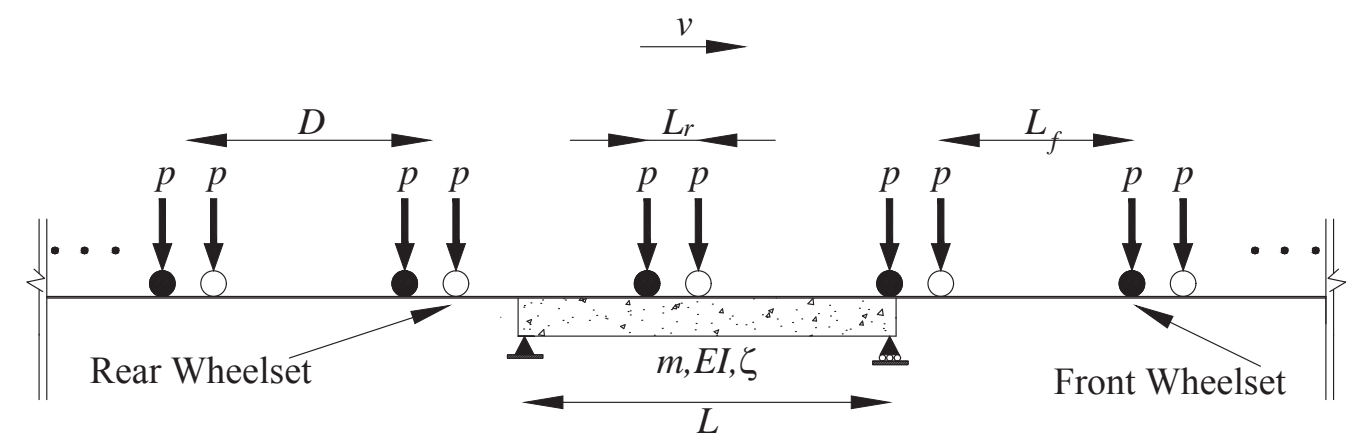

(b) Simplified adopted model

Figure 2: General modeling of railway bridge under passage of trains versus simplified adopted load model

$$
\begin{gathered}
\ddot{q}_{n}(t)=\frac{2 p L^{3}}{E I \pi^{4}}\left[P_{n}(v, t)+P_{n}\left(v, t-L_{f} / v\right)\right] \\
p_{1}=1-S_{n}{ }^{2}, \quad p_{2}=2 \xi_{n} S_{n}, \quad p_{3}=-\xi_{n} \omega_{n} \\
p_{4}=\frac{S_{n}\left(2 \xi_{n}{ }^{2}+S_{n}{ }^{2}-1\right)}{\sqrt{1-\xi_{n}{ }^{2}}, \quad t_{k}=\frac{(k-1) D}{v}} \\
P_{n}(v, t)=\frac{1}{n^{4}} \sum_{k=1}^{N} \frac{1}{p_{1}{ }^{2}+p_{2}{ }^{2}}\left[A\left(t-t_{k}\right) \cdot H\left(t-t_{k}\right)+(-1)^{n+1} A\left(t-t_{k}-\frac{L}{v}\right) \cdot H\left(t-t_{k}-\frac{L}{v}\right)\right] \\
\alpha_{1}=-p_{1} \Omega_{n}{ }^{2}, \quad \alpha_{2}=p_{2} \Omega_{n}{ }^{2}, \quad \alpha_{3}=p_{2} p_{3}{ }^{2}+2 p_{3} p_{4} \omega_{d n}-p_{2} \omega_{d n}{ }^{2} \\
\alpha_{4}=p_{3}{ }^{2} p_{4}-2 p_{2} p_{3} \omega_{d n}-p_{4} \omega_{d n}{ }^{2} \\
A(t)=\alpha_{1} \sin _{n} t+\alpha_{2} \cos \Omega_{n} t+e^{p_{3} t}\left[\alpha_{3} \cos \omega_{d n} t+\alpha_{4} \sin \omega_{d n} t\right]
\end{gathered}
$$


Where, $\ddot{u}(t)$ is the deck acceleration, $\ddot{q}_{n}(t)$ is the generalized coordinate, $\phi_{n}(x)$ is the mode shape of the $n^{\text {th }}$ mode of vibration, $L$ is the span length of the beam, $p$ is the axle load, $E I$ is the flexural rigidity of the beam, $v$ is the speed of the train, $L_{f}$ is the distance between the front and rear wheelsets subsystems in each car body, $S_{n}=n \pi v / \omega_{n} L$ is the speed parameter corresponding to the $n^{\text {th }}$ mode of vibration, $\xi_{n}$ is the damping ratio of the $n^{t h}$ mode of vibration, $D$ is the distance between front to front or rear to rear wheelsets in subsequent car bodies, $N$ is the number of car bodies, $H(t)$ is the Heaviside function, $\Omega_{n}=S_{n} \omega_{n}$ is the exciting frequency implied by the moving load and $\omega_{d n}=\omega_{n} \sqrt{1-\xi_{n}^{2}}$ is the damped frequency of the $n^{\text {th }}$ mode of vibration.

It is worthwhile noting that only the first mode of vibration is considered here for the sake of simplification. Such consideration imposes some errors particularly at offresonance speeds; however, it results in accurate enough predictions for resonance speeds. Therefore, considering only one mode of vibration may not generally affect the safety of the bridge.

Additionally, it has been previously observed that spreading axle loads through the track structure (rails, sleepers and ballast) significantly reduces the bridge response particularly for the short span ones [11]. Therefore, a reduction coefficient is multiplied by the obtained response from the aforementioned analytical solution; which is obtained by fitting the presented relationship in Eq. 11 to the graphically proposed reduction factor in $[12]$.

$$
R(\lambda)=\left\{\begin{array}{cc}
0.14 & , \lambda \leq 1.5 \\
-0.00011 \lambda^{4}+0.0053 \lambda^{3}-0.083 \lambda^{2}+0.57 \lambda-0.54 & , 1.5<\lambda<8.0 \\
1.0 & , \lambda \geq 8.0
\end{array}\right.
$$

Where, $\lambda=v / f_{1}$ is the wavelength.

\subsection{Verification of analytical solution}

A previously presented example by Yang et al. [9] is taken into account to verify the adopted analytical solution. For this objective, mid-span acceleration of a simply supported beam with $L=20 \mathrm{~m}, I=2.81 \mathrm{~m}^{4}, E=29.43 \mathrm{GPa}$ and $m=34088 \mathrm{~kg} / \mathrm{m}$ under passage of a train with $N=5, L_{f}=18 \mathrm{~m}, L_{r}=6 \mathrm{~m}, p=215.6 \mathrm{kN}$ and $v=$ $122.4 \mathrm{~km} / \mathrm{h}$ is calculated. The considered speed corresponds to the resonance speed of the bridge. Additionally, two cases, i.e. without damping and $\xi=2.5 \%$ is taken into account. The obtained analytical accelerations are compared with those resulted from numerical evaluations in Figure 5; which, a promising agreement can be distinguished. It is worthwhile noting that the numerical calculations are conducted using Newmark's average acceleration method; however, reduction factor due to load distributions is neglected for the comparison objectives.

\section{BASIC VARIABLES AND LIMIT STATE FUNCTION}

As mentioned, the current article aims at evaluating the probability of ballast instability under passage of high-speed trains. Thus, the performance function regarding this limit state would be as follows:

$$
\begin{array}{r}
G(\mathbf{X})=a_{\text {limit }}-\left|a_{\text {max }}(\mathbf{X})\right|\left(1+\chi_{m}\right) \\
\mathbf{X}=\left(N, D, p, v, L, E, I, A_{d}, A_{b}, \rho_{d}, \rho_{b}, \xi, \chi_{m}\right)
\end{array}
$$




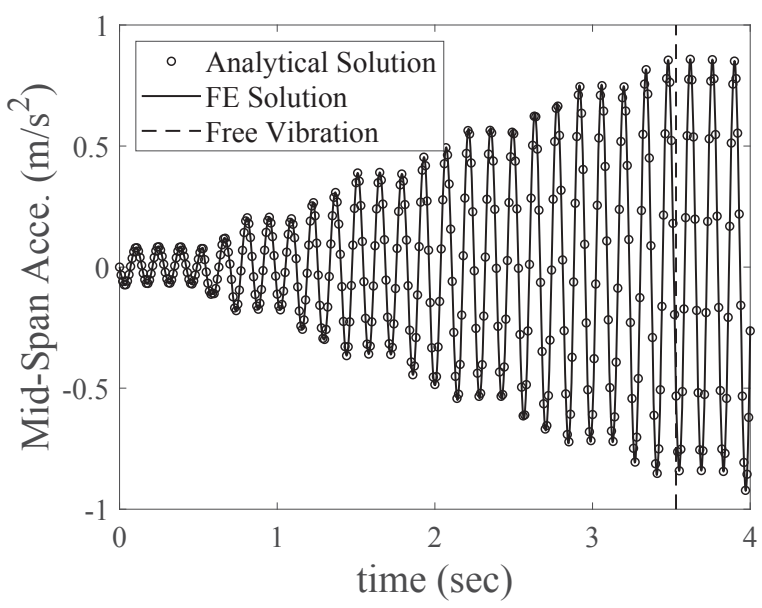

(a) Without damping

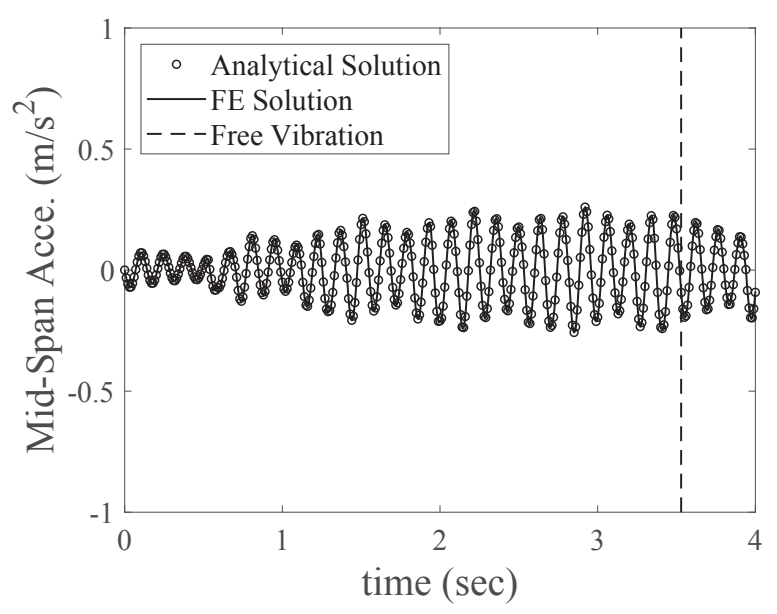

(b) $\xi=2.5 \%$

Figure 3: Verification of analytical solution with numerical method by comparing mid-span acceleration

Where, $a_{\text {limit }}$ is the vertical acceleration initiating ballast instability, $E$ is the modulus of elasticity of deck, $I$ is the moment of inertia of deck, $A_{c}$ is the deck area, $A_{b}$ is the ballast area, $\rho_{d}$ is the mass density of deck, $\rho_{b}$ is the mass density of ballast, $\xi$ is the damping ratio and $\chi_{m}$ is the model uncertainty. It is worthwhile noting that ballast has much lower modulus of elasticity than the deck [1]; which led its contribution in stiffness to be neglected.

As previously mentioned, only few shaking table tests have been conducted to determine the vertical acceleration leading ballast instability [4]. Thus, a triangular probability distribution is considered for $a_{\text {limit }}$; which its mode equals to experimentally captured value, i.e. $0.7 \mathrm{~g}$. Furthermore, a possible variation of $0.1 \mathrm{~g}$ is taken into account based on authors' judgment to define its boundaries.

Regarding the applied loads, it is hardly possible to collect information corresponding to the variability of parameters in commercial trains. Thus, proposed load models in Annex E of Eurocode [13] are applied here. Hence, the uncertainty on moving loads would be a function of constant axle load $\left(p_{\text {axle }}\right)$ and occupancy rate ( $\alpha$ in \%). By assuming the full passenger capacity of each car body as 65 and average weight of each passenger as $70 \mathrm{~kg}$, the moving load can be calculated as Eq. 13; which is developed in $\mathrm{kN}$ units.

$$
p=p_{\text {axle }}+0.225 \alpha
$$

In order to select practically reasonable geometrical values (i.e. span length, moment of inertia and deck/ballast area) 62 single span and single track bridges in Sweden are surveyed. Detailed information about them can be found in [14]. The span length of the desired bridges varies between [5-30] m. Therefore, evaluations are conducted within this range for deterministic span lengths with a resolution of $5 \mathrm{~m}$.

The distribution of span length, moment of inertia, deck and ballast areas in addition to their linear correlations are depicted in Figure 4. A strong correlation between span length, moment of inertia and deck area is evident; while as expected, the ballast area seems to be independent on span length. Hence, a conditional probability for the moment of inertia and deck area is assigned (see Figures 5a and 5b); which, the parameters are extracted by regression analysis and fitting lower and upper boundaries. On the other 
hand, the shown scatter plot of the ballast area (see Figure 5c) reveals that they mostly vary around mean value regardless of the span length. Due to extracting distributions from a limited database, a triangular probability distribution is adopted for the all geometrical random variables.

It is well-known that the response of the bridges is highly dependent on the damping ratio particularly around resonant speed. Additionally, the contribution of several factors makes damping measurement a tedious task; which may result in scattered outcomes. Therefore, the proposed lower bound in [12] is taken here as the mean value of the damping ratio.

Discussed simplifications and existing lack of knowledge on different aspects of the problem deviate the predicted responses by the adopted solution from corresponding real values. Thus, a model uncertainty $\left(\chi_{m}\right)$ is applied to the limit state function; which should be obtained from comparison of the experimentally captured values with those resulted from the numerical model. However, the lack of such information led to propose a model based on the authors' judgment. In this regard, a Gaussian probability density function with zero mean and a standard deviation of $20 \%$ is taken into account.

Considering all, the assigned parameters for the variables are reported in Table 1.

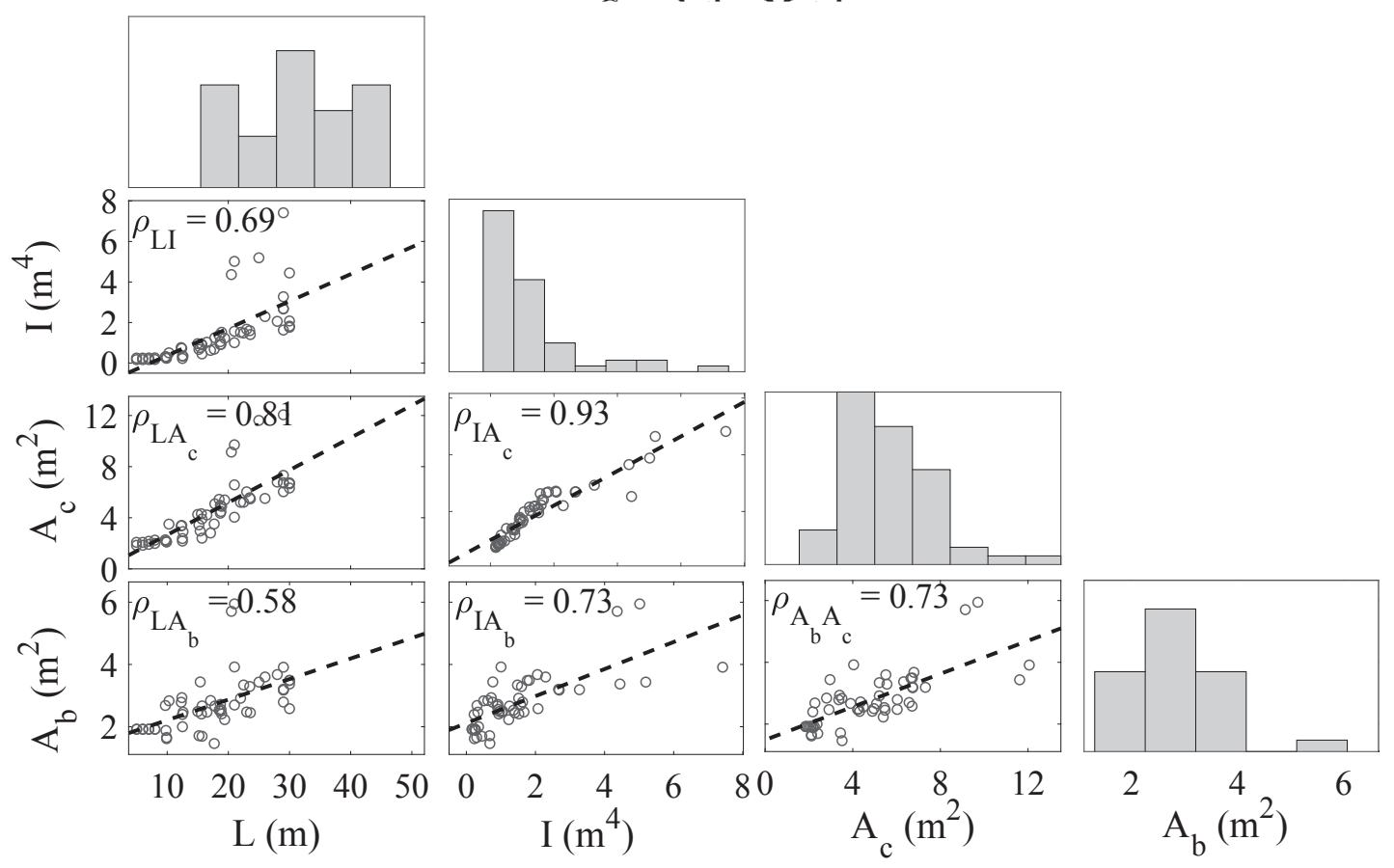

Figure 4: Correlation between span length, deck moment of inertia, deck area and ballast area

\section{RESULTS AND DISCUSSION}

Initially, the considered bridges are assessed following deterministic approaches. For this objective, the speed of passing trains swept between $100 \mathrm{~km} / \mathrm{h}$ up to $400 \mathrm{~km} / \mathrm{h}$; while the average values are assigned for each variable. The analyses are repeated by changing each variable to mean plus/minus one standard deviation; while other variables remained constant. Hence, the train speed leading the vertical acceleration to surpass $3.5 \mathrm{~m} / \mathrm{s}^{2}$ is extracted (see for instance Figure 6). The obtained deterministic operating train speeds are reported in Table 2. 


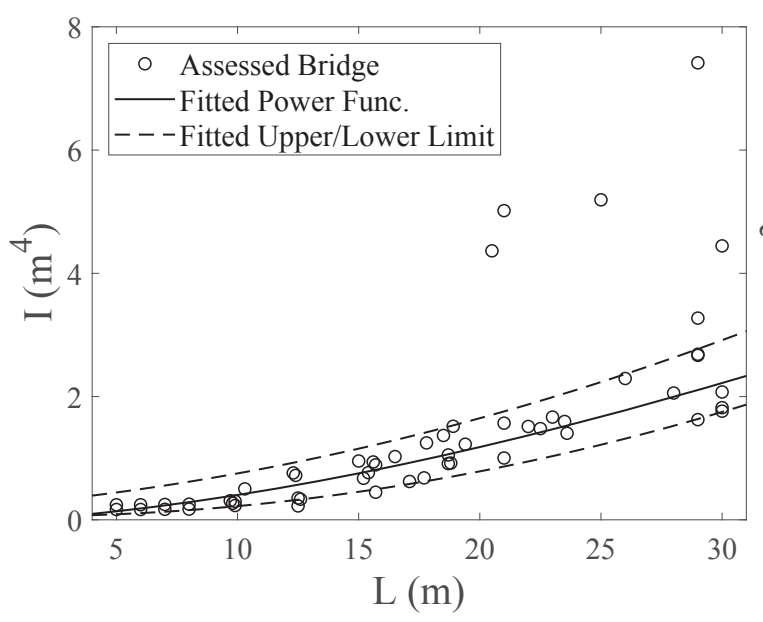

(a) Deck moment of inertia

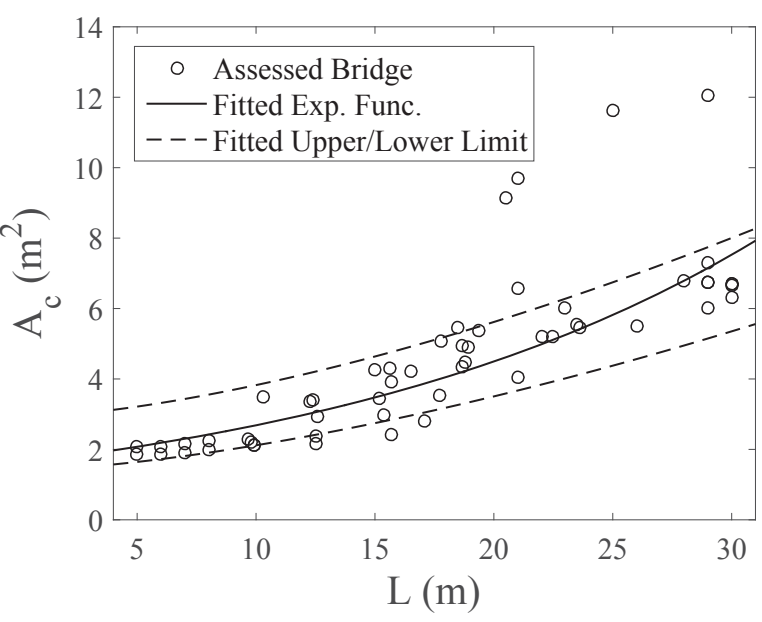

(b) Deck cross section area

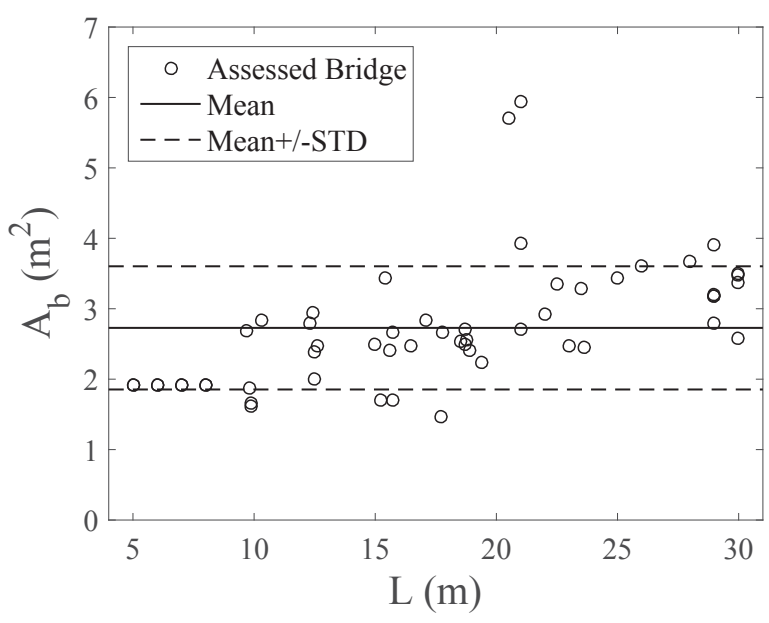

(c) Ballast area

Figure 5: Distribution function for deck moment of inertia, deck cross section area and ballast area as a function of span length

Then, the reliability of the developed limit state function is assessed using FORM. In this regard, UQLab toolbox [16] is employed to conduct the analyses and extract the corresponding probability of violence. For this objective, the span length of the bridge is assumed as a deterministic parameter varying in the desired range and properties of the other parameters are adopted as those tabulated in Table 1. Then, the reliability of each bridge is assessed for all considered trains with different configurations; while a speed resolution of $2 \mathrm{~km} / \mathrm{h}$ is taken into account. Since the probability of violation is calculated at discrete points; then, the average exceeding (failure) probability $\left(p_{f, a v e}\right)$ can be calculated using Eq. 14 [18].

$$
p_{f, a v e}=\int_{v_{l}}^{v_{u}} P\left(a_{\text {deck }}(v)>a_{\text {limit }}\right) f_{v}(v) d v \approx \frac{1}{n} \sum_{i=1}^{n} P\left(a_{\text {deck }}\left(v_{i}\right)>a_{\text {limit }}\right)
$$

Where, $n$ is the number of discrete evaluations. 


\begin{tabular}{|c|c|c|c|c|c|}
\hline Variable & Unit & Dist. & $\begin{array}{c}\text { Mean/Mode } \\
\text { Min. }\end{array}$ & $\begin{array}{l}\text { STD/Bound. } \\
\text { Max. }\end{array}$ & Ref. \\
\hline$a_{\text {limit }}$ & $\mathrm{m} / \mathrm{s}^{2}$ & Triangular & 7.0 & {$[6.0-8.0]$} & - \\
\hline$N$ & - & Uniform (Integer) & 11 & 18 & {$[13]$} \\
\hline$D$ & $\mathrm{~m}$ & Uniform & 18 & 27 & [13] \\
\hline$L_{r}$ & $\mathrm{~m}$ & Uniform & 2.5 & 3.5 & {$[13]$} \\
\hline$p_{\text {axle }}$ & $\mathrm{kN}$ & Deterministic & 170 & - & [13] \\
\hline$\alpha$ & $\%$ & Uniform & 0 & 100 & - \\
\hline$v$ & $\mathrm{~km} / \mathrm{h}$ & Deterministic & 100 & 400 & - \\
\hline$L$ & $\mathrm{~m}$ & Deterministic & 5 & 30 & - \\
\hline$E$ & $\mathrm{GPa}$ & Truncated Gaussian $^{a}$ & 29.7 & 3.5 & {$[15]$} \\
\hline$I$ & $\mathrm{~m}^{4}$ & Triangular ${ }^{b}$ & $\mathrm{M}_{I}$ & {$\left[\mathrm{LB}_{I}-\mathrm{UB}_{I}\right]$} & - \\
\hline$A_{c}$ & $\mathrm{~m}^{2}$ & Triangular ${ }^{c}$ & $\mathrm{M}_{A_{c}}$ & {$\left[\mathrm{LB}_{A_{c}}-\mathrm{UB}_{A_{c}}\right]$} & - \\
\hline$A_{b}$ & $\mathrm{~m}^{2}$ & Triangular & 2.7 & {$[1.85-3.6]$} & - \\
\hline$\rho_{c}$ & $\mathrm{~kg} / \mathrm{m}^{3}$ & Lognormal $^{d}$ & 7.82 & 0.04 & {$[1]$} \\
\hline$\rho_{b}$ & $\mathrm{~kg} / \mathrm{m}^{3}$ & Uniform & 1500 & 2100 & {$[1]$} \\
\hline$\xi$ & $\%$ & $\operatorname{Lognormal~}^{e}$ & $\mu_{\xi}$ & $\sigma_{\xi}$ & {$[1,12]$} \\
\hline$\chi_{m}$ & $\%$ & Gaussian & 0 & 20 & - \\
\hline
\end{tabular}

a The mean value is obtained from $5015 \sqrt{f_{c}^{\prime}}[\mathrm{MPa}]$ and considering C35 as typical concrete class in bridges. A COV of equaling $12 \%$ is also considered. Due to controlled construction conditions of the railway bridges, the assigned probability distribution is truncated for values deviated more than two standard deviation from the mean value.

$b$ The mode is obtained from regression analysis between span length and moment of inertia in the surveyed bridges; which equals as $M_{I}=0.011\left(L^{1.55}\right)$. Furthermore, the lower and upper bounds would obtain from $\mathrm{LB}_{I}=0.002 L^{2}-0.004 L+0.06$ and $\mathrm{UB}_{I}=0.00185 L^{2}+0.034 L+0.23$, respectively.

$c$ The mode is obtained from regression analysis between span length and cross section area; which equals as $M_{A_{c}}=1.6 \exp (0.052 L)$. Since cross section area is highly correlated to the moment of inertia; therefore, boundaries are obtained from $\mathrm{LB}_{A_{c}}=1.781 M_{I}+1.4$ and $\mathrm{UB}_{A_{c}}=2.3 M_{I}+2.9$, respectively.

${ }^{d}$ Reported mean and standard deviation correspond to $2500 \mathrm{~kg} / \mathrm{m}^{3}$ and $100 \mathrm{~kg} / \mathrm{m}^{3}$ mean and standard deviation in physical space, respectively.

$e$ The mean value of the damping in normal space is obtained from $1.5+0.07(20-L)[12]$ for bridges with less than $20 \mathrm{~m}$ span length and equals to $1.5 \%$ [12] for others. It is needed to be transformed to logarithmic space. Furthermore, the standard deviation of the lognormal distribution should be obtained by considering aforesaid mean and standard deviation of $0.3 \%$ in physical space.

Table 1: Considered variables and their stochastic/deterministic properties

The aforesaid probabilities are required to be compared by an appropriate target reliability index. Regarding the nature of the problem, it can be inferred both as serviceability (SLS) or ultimate (ULS) limit states. The former corresponds to the maintenance and tamping of the ballast; however, the later can be considered as severe cases in which ballast instability may lead to derailment. Hence, $\beta=3.1\left(p_{f} \approx 10^{-3}\right)$ is considered as the target reliability $[17,18]$.

Then, the average probability of exceeding vertical limit acceleration versus train speed is illustrated in Figure 7. Only outcomes corresponding to $5 \mathrm{~m}$ and $30 \mathrm{~m}$ span lengths are presented here for the sake of brevity; while, the extracted operating speeds for all bridges 


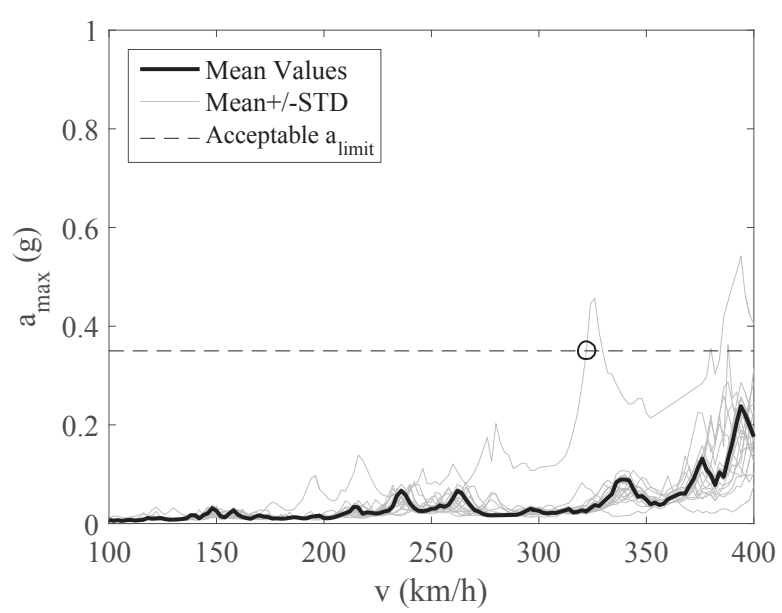

(a) $\mathrm{L}=5 \mathrm{~m}$

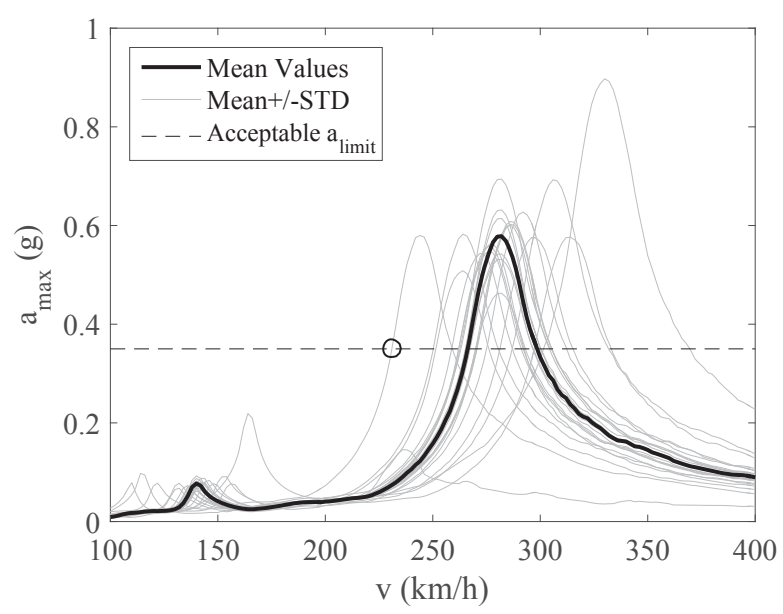

(b) $\mathrm{L}=30 \mathrm{~m}$

Figure 6: Deterministic running safety assessment of the considered bridges

are reported in Table 2. As can be seen, the conventional deterministic approach does not lead at a constant safety level; which may result in designs with ambiguous capacity. Furthermore, it can be inferred from those safety indices that the conventional design method does not satisfy the desired safety level $\left(p_{f}=10^{-3}\right)$. The latter statement is particularly true for bridges with larger span lengths. Such lower safety may arise from several factors. Most importantly, shorter span bridges have generally higher frequencies. Thus, higher train speeds are required to observe the resonance phenomenon. In addition, the distribution of loads on track structure significantly reduces the response of bridges with shorter span lengths; while its influence would be negligible for larger span lengths.

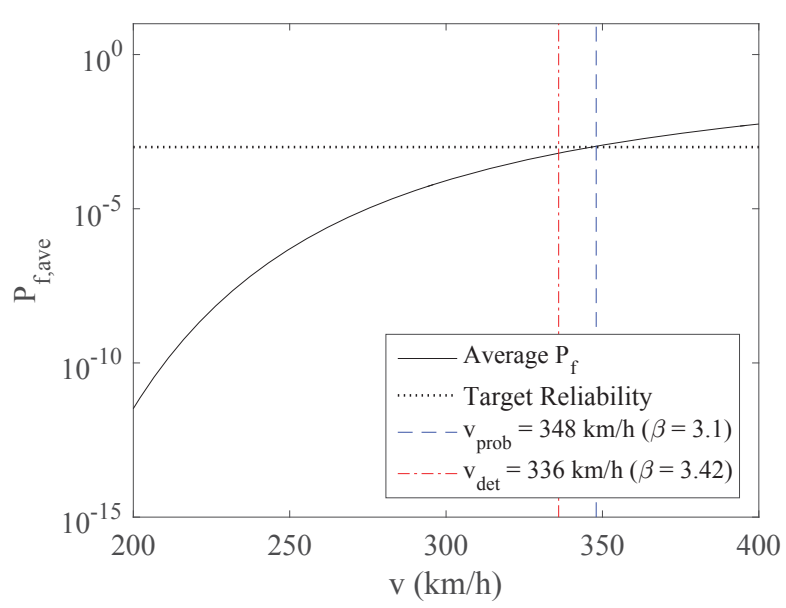

(a) $\mathrm{L}=5 \mathrm{~m}$

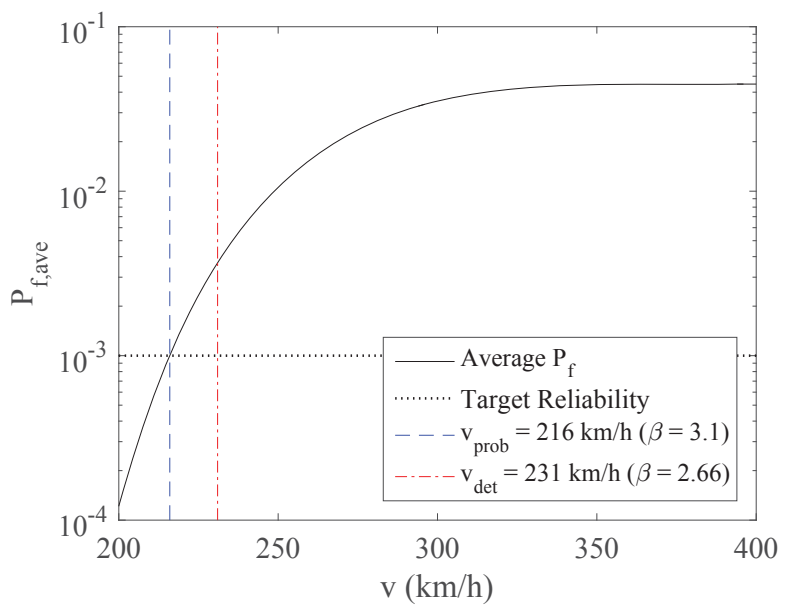

(b) $\mathrm{L}=30 \mathrm{~m}$

Figure 7: Reliability-based assessment of the considered bridges

Finally, the sensitivity of the reliability evaluations to the considered random variables is assessed using both direction cosines and omission sensitivity factor (see Figure 8). As can be seen, the response is most sensitive to variations on car-body length, modulus 


\begin{tabular}{cccc}
\hline $\begin{array}{c}L \\
(\mathrm{~m})\end{array}$ & $\begin{array}{c}v_{\text {prob }} \\
(\mathrm{km} / \mathrm{h})\end{array}$ & $\begin{array}{c}v_{\text {det }} \\
(\mathrm{km} / \mathrm{h})\end{array}$ & $\beta_{\text {det }}$ \\
\hline 5 & 348 & 322.0 & 3.42 \\
10 & 230 & 315.5 & 1.97 \\
15 & 250 & 284.0 & 2.37 \\
20 & 170 & 215.0 & 2.01 \\
25 & 148 & 186.0 & 2.27 \\
30 & 216 & 231.0 & 2.66 \\
\hline
\end{tabular}

Table 2: Comparison between reliability-based assessment versus deterministic evaluation

of elasticity and deck moment of inertia. It is worthwhile to note that deck/ballast cross section area and their mass densities are also relatively important; which leads the reliability level to be sensitive on their combination, i.e. mass of the bridge.

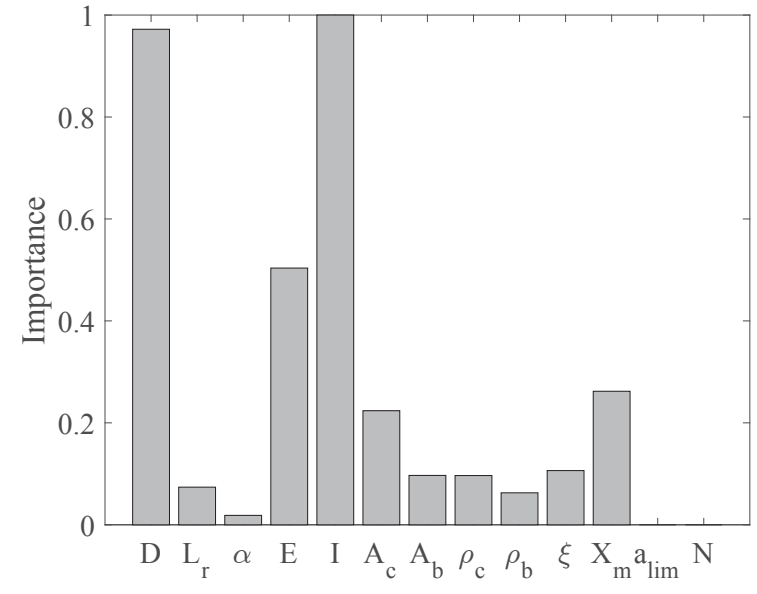

(a) Sensitivity from direction cosines

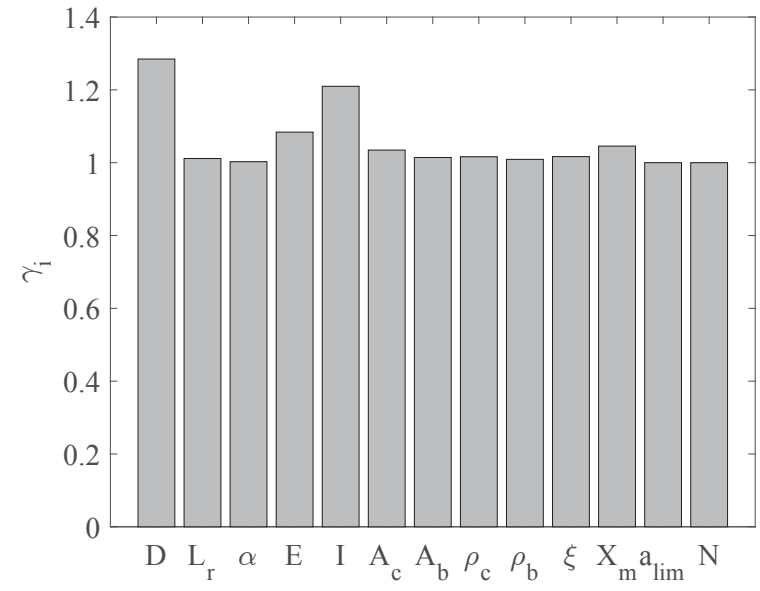

(b) Omission sensitivity

Figure 8: Sensitivity of the reliability evaluations to the considered random variables

\section{CONCLUSIONS}

- An existing closed-form solution is adopted to evaluate the reliability of simply supported single span high-speed railway bridges using FORM. It was noted that the conventional deterministic dynamic assessments result in bridges which do not satisfy desired target reliability level $(\beta=3.1)$.

- Despite applying a safety factor of 2.0 for the vertical acceleration limit, the conventional design method does not lead to a constant safety level on bridges with different span lengths.

- Reliability of the bridges are mostly sensitive to the car-body length, modulus of elasticity and deck moment of inertia. Moreover, the mass of the bridge would be a function of deck cross section area, ballast area, concrete mass density and ballast mass density. Thus, by considering their importance the assessed reliability would be considerably sensitive to their combination, i.e. mass of the bridge. 


\section{REFERENCES}

[1] Rocha J.M., Probabilistic Methodologies For the Safety Assessment of Short Span Railway Bridges For High-Speed Traffic, PhD Dissertation, University of Porto, 2015.

[2] Arvidsson T., Train-Track-Bridge Interaction for the Analysis of Railway Bridges and Train Running Safety, PhD Dissertation, KTH Royal Institute of Technology, 2018.

[3] Frýba L., A Rough Assessment of Railway Bridges For High Speed Trains, Engineering Structures, 23, 548-556, 2001.

[4] Zacher M., Baeßler M., Dynamic Behaviour of Ballast on Railway Bridges, in Dynamics of High-Speed Railway Bridges, Taylor \& Francis, 2009.

[5] CEN, EN 1991-A2, Eurocode 0: Basis of Structural Design - Annex A2: Applications for Bridges, European Committee for Standardization, 2005.

[6] Melchers R.E., Beck A.T., Structural Reliability Analysis And Prediction, Wiley, ISBN: 978-1-119-26599-3, 2018.

[7] Sørensen J.D., Notes in Structural Reliability Theory And Risk Analysis, Department of Civil Engineering, Aalborg University, 2011.

[8] Madsen H., Omission Sensitivity Factors, Structural Safety, 5, 35-45, 1988.

[9] Yang Y.B., Yau J.D., Hsu L.C., Vibration of Simple Beams Due to Trains Moving at High Speeds, Engineering Structures, 19(11), 936-944, 1997.

[10] Yang Y.B., Yau J.D., Wu Y.S., Vehicle-Bridge Interaction Dynamics With Applications to High-Speed Railways, World Scientific, ISBN: 981-238-847-8, 2004.

[11] Jin Z., Huang B., Ren J., Pei S., Reduction of Vehicle-Induced Vibration of Railway Bridges due to Distribution of Axle Loads Through Track, Shock and Vibration, Article ID 2431980, 2018.

[12] ERRI D 214/RP 9, Rail Bridges for Speeds > $200 \mathrm{~km} / \mathrm{h}$, Final Report Part A: Synthesis of the Results of D 214 Research, European Rail Research Institute, 1999.

[13] CEN, EN 1991-2, Eurocode 1: Actions on Structures - Part 2: Traffic Loads on Bridges, European Committee for Standardization, 2003.

[14] Museros P., Andersson A., Marti V., Karoumi R., Dynamic Behaviour of Bridges Under Critical Articulated Trains: Signature and Bogie Factor Applied to the Review of Some Regulations Included in EN 1991-2, Submitted to Journal of Rail and Rapid Transit, 2020 (Under Review).

[15] Wiśniewski D.F., Safety Formats For the Assessment of Concrete Bridges with Special Focus on Precast Concrete, PhD Dissertation, University of Minho, 2007.

[16] Marelli S., Sudret B., UQLab: A Framework for Uncertainty Quantification in MAT$L A B$, In the 2nd International Conference on Vulnerability and Risk Analysis and Management (ICVRAM 2014), University of Liverpool, UK, 2014. 
[17] JCSS, Probabilistic Model Code, 12th Draft, JCSS-Joint Committee on Structural Safety, ISBN: 978-3-909386-79-6.

[18] Hirzinger B., Adam C., Oberguggenberger M., Salcher P., Approaches for Predicting the Probability of Failure of Bridges Subjected to High-Speed Trains, Probabilistic Engineering Mechanics, 59, 103021, 2020. 